\title{
TITLE:
}

\section{Initial nucleation of AlN grown directly on sapphire substrates by metal-organic vapor phase epitaxy}

\section{$\operatorname{AUTHOR}(S)$ :}

Banal, Ryan G.; Funato, Mitsuru; Kawakami, Yoichi

\section{CITATION:}

Banal, Ryan G.... [et al]. Initial nucleation of AIN grown directly on sapphire substrates by metal-organic vapor phase epitaxy. APPLIED PHYSICS LETTERS 2008, 92(24): 241905.

\section{ISSUE DATE:}

2008-01-16

URL:

http://hdl.handle.net/2433/84582

\section{RIGHT:}

Copyright 2008 American Institute of Physics. This article may be downloaded for personal use only. Any other use requires prior permission of the author and the American Institute of Physics. 


\title{
Initial nucleation of AIN grown directly on sapphire substrates by metal-organic vapor phase epitaxy
}

\author{
Ryan G. Banal, Mitsuru Funato, and Yoichi Kawakamia \\ Department of Electronic Science and Engineering, Kyoto University, Kyoto 615-8510, Japan
}

(Received 26 March 2008; accepted 8 May 2008; published online 17 June 2008)

\begin{abstract}
AlN layers were grown directly on sapphire (0001) substrates using three different growth sequences based on metal-organic vapor phase epitaxy with an emphasis on initial nucleation processes. These three methods were simultaneous, alternating supply of aluminum and nitrogen sources, and a combination of the two. In all the methods, nucleation was initiated by three-dimensional (3D) islands with a typical diameter of $\sim 20 \mathrm{~nm}$. Enhanced migration by the alternating source supply caused highly 3D AlN ridge structures at the sapphire molecular steps. These ridge structures prevented a flattened AlN surface and, in addition, moderated lattice relaxation, suggesting the importance of controlling the initial nucleation in determining the film's properties. In fact, the hybridized method, derived from the simultaneous and alternating supply methods, was able to control the initial nucleation, and provided the best film quality; the 600 -nm-thick AlN grown by this method had an atomically flat surface free of pits and particles, and the x-ray diffraction line widths were $\sim 45$ and $\sim 250$ arcsec for the (0002) and (1012) planes, respectively. (C) 2008 American Institute of Physics. [DOI: 10.1063/1.2937445]
\end{abstract}

AIN has enormous potential for UV and deep-UV optical and high-power electronic devices due to its hightemperature stability and thermal conductivity. Currently the absence of defect-free bulk substrates has led to AIN being grown on foreign substrates such as sapphire and $\mathrm{SiC}^{1-3}$ It is well known that heteroepitaxial growth generates highdensity threading dislocations, which consequently affect the radiative property of a device. ${ }^{4}$ Although an emission at $210 \mathrm{~nm}$ under current injection has been demonstrated, the external quantum efficiency remains extremely low $\left(10^{-6} \%\right)$ due to the high dislocation density. ${ }^{5}$ Therefore, an AlN layer with a smooth surface and a low defect density is a prerequisite to realize high-efficiency devices.

To obtain high-quality AlN, several growth methods have been proposed. Although high-temperature growth at $1400{ }^{\circ} \mathrm{C}$ has been demonstrated to considerably improve film quality, ${ }^{6}$ issues such as difficulty in impurity doping may pose problems. Another option is an alternating supply of group III and V source precursors, which enables growth at $\sim 1200{ }^{\circ} \mathrm{C} .^{7-14}$ Migration-enhanced epitaxy (MEE) has realized high-quality $\mathrm{AlN}$ by enhancing $\mathrm{Al}$ adatom migration and consequent lateral growth. ${ }^{12,14}$ The full width at half maximum (FWHM) of a (0002) x-ray diffraction (XRD) $\omega$-scan can be as narrow as 50 arcsec. ${ }^{12} \mathrm{~A}$ modified MEE, which is characterized by a combination of simultaneous source supply and conventional MEE, has also been proposed. $^{10,13}$ In addition to source flow sequences, the nucleation layer appears to be a key factor for obtaining high-quality epilayers. In fact, low-temperature ${ }^{12,14}$ (LT) and high-temperature ${ }^{10,13}$ AlN have been employed, although some research groups did not use either of these. ${ }^{1,6}$ Such a fragmentary understanding of the AlN growth is, we believe, largely due to the lack of investigations on the initial stages of growth. Therefore, in this letter, we examined the initial nucleation and lattice relaxation processes of several growth methods, and correlated them to the epilayer quality.

${ }^{a)}$ Electronic mail: kawakami@kuee.kyoto-u.ac.jp.
AlN epilayers were grown on sapphire (0001) substrates using metal-organic vapor phase epitaxy (MOVPE). The nucleation layers were not incorporated for the reason described below. The total pressure was maintained at 76 Torr. Source gases were trimethylaluminum (TMA) and $\mathrm{NH}_{3}$, and the optimized V/III ratio was $\sim 170$. Prior to growth, the sapphire substrates were thermally etched in $\mathrm{H}_{2}$ for $10 \mathrm{~min}$ at $1220{ }^{\circ} \mathrm{C}$, resulting in surfaces with monolayer steps and $\sim 360$-nm-wide atomically flat terraces. Then the AlN layers were grown at $1200{ }^{\circ} \mathrm{C}$ by MEE, modified MEE, or simultaneous source supply method, as shown in Fig. 1. For conventional MEE [Fig. 1(a)], TMA and $\mathrm{NH}_{3}$ were each supplied in $1 \mathrm{~s}$ durations $(2 \mathrm{~s} /$ cycle). For modified MEE [Fig. 1(b)], a simultaneous supply for $1 \mathrm{~s}$ was inserted in the above-mentioned conventional MEE cycle, and thus, one cycle of this method $(=3 \mathrm{~s})$ consisted of one cycle $(=2 \mathrm{~s})$ of MEE and $1 \mathrm{~s}$ of simultaneous supply. Except for the simultaneous supply method, $\mathrm{NH}_{3}$ was initially introduced into the reactor for $1 \mathrm{~s}$. As indicated by the $20 \% \mathrm{KOH}$ solution at $70{ }^{\circ} \mathrm{C}$, the grown films had the $\mathrm{Al}$ polarity.

Figure 1 shows the results of surface analyses by atomic force microscopy (AFM) for 600-nm-thick AlN layers grown by these three methods. Owing to the optimized growth conditions, all three AlN layers exhibited atomically smooth surfaces. However, detailed observations revealed that MEEgrown AlN involved pits with a diameter of $\sim 10-50 \mathrm{~nm}$, especially along the step edge [Fig. 1(a)]. These pits are attributed to incomplete island coalescence, as discussed below. Although the simultaneous supply method yielded much larger pits and $\mathrm{Al}$ particles [Fig. 1(c)], the modified MEE achieved an atomically smooth surface free of defects [Fig. 1(b)]. These morphological characteristics resulted in the

TABLE I. FWHM values of (0002) and (1012) $\omega$-scans.

\begin{tabular}{lccc}
\hline \hline $\operatorname{arcsec}$ & MEE & Modified MEE & Simultaneous \\
\hline$(0002)$ & 45.1 & 42.8 & 52.6 \\
$(10 \overline{1} 2)$ & 1443.5 & 244.5 & 889.1 \\
\hline \hline
\end{tabular}


(a) MEE
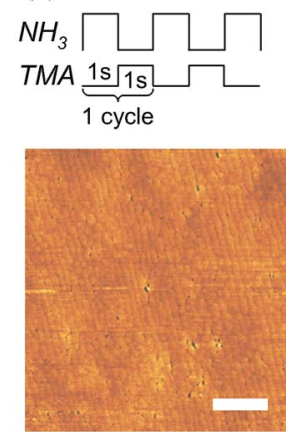

FIG. 1. (Color online) Growth sequences and surface morphologies of $\sim 600$-nm-thick AlN grown by (a) conventional MEE, (b) modified MEE, and (c) simultaneous supply method. Scale bar represents $1 \mu \mathrm{m}$.

root-mean-square roughness of $0.12 \mathrm{~nm}$ for modified MEE, $0.35 \mathrm{~nm}$ for MEE, and $0.52 \mathrm{~nm}$ for the simultaneous supply. The AlN quality was further confirmed by XRD symmetric (0002) and asymmetric (10 $\overline{1} 2) \omega$-scans. Table I tabulates the results. Regardless of the preparation method, all AlN had

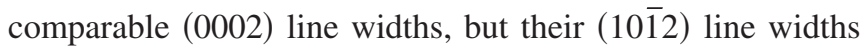
were widely scattered, suggesting similar screw dislocation densities, but vastly different edge dislocation densities. The estimated screw dislocation component in modified-MEEgrown AlN was $\sim 4.0 \times 10^{6} \mathrm{~cm}^{-2}$ from the (0002) FWHM of 43 arcsec. $^{15}$

The growth rates were estimated to be $0.22 \mathrm{~nm} /$ cycle for conventional MEE, $0.70 \mathrm{~nm} /$ cycle for modified MEE, and $0.50 \mathrm{~nm} / \mathrm{s}$ for the simultaneous supply. Because a modifiedMEE cycle consisted of one cycle of MEE and $1 \mathrm{~s}$ of a simultaneous supply, the good coincidence between 0.70 $\mathrm{nm} /$ cycle (modified MEE) and the sum of $0.22 \mathrm{~nm} /$ cycle (MEE) and $0.50 \mathrm{~nm} / \mathrm{s}$ (simultaneous supply) validates the assumption that the growth behavior during modified MEE is well approximated by a sequential, independent appearance of those during MEE and the simultaneous supply method. Because their growth rates did not change when AlN was thicker than $40 \mathrm{~nm}$, this assumption is reasonable even in the initial growth. Moreover, the relatively faster growth rate by the simultaneous supply method than that by MEE implies the absence of premature reactions.

To clarify the above-mentioned difference in the overall AlN quality due to the growth methods, the initial nucleation processes were investigated. Figure 2 displays the AFM images for AlN with nominal thicknesses of $\sim 2$ and $14 \mathrm{~nm}$ just after the initial nucleation as well as for thicker AlN just after the coalescence of the initial nuclei. Regardless of the growth method, the initial nuclei were three-dimensional (3D) islands with a typical diameter of $\sim 20 \mathrm{~nm}$. As theoretically expected, ${ }^{16} 3 \mathrm{D}$ growth is more favorable than two-
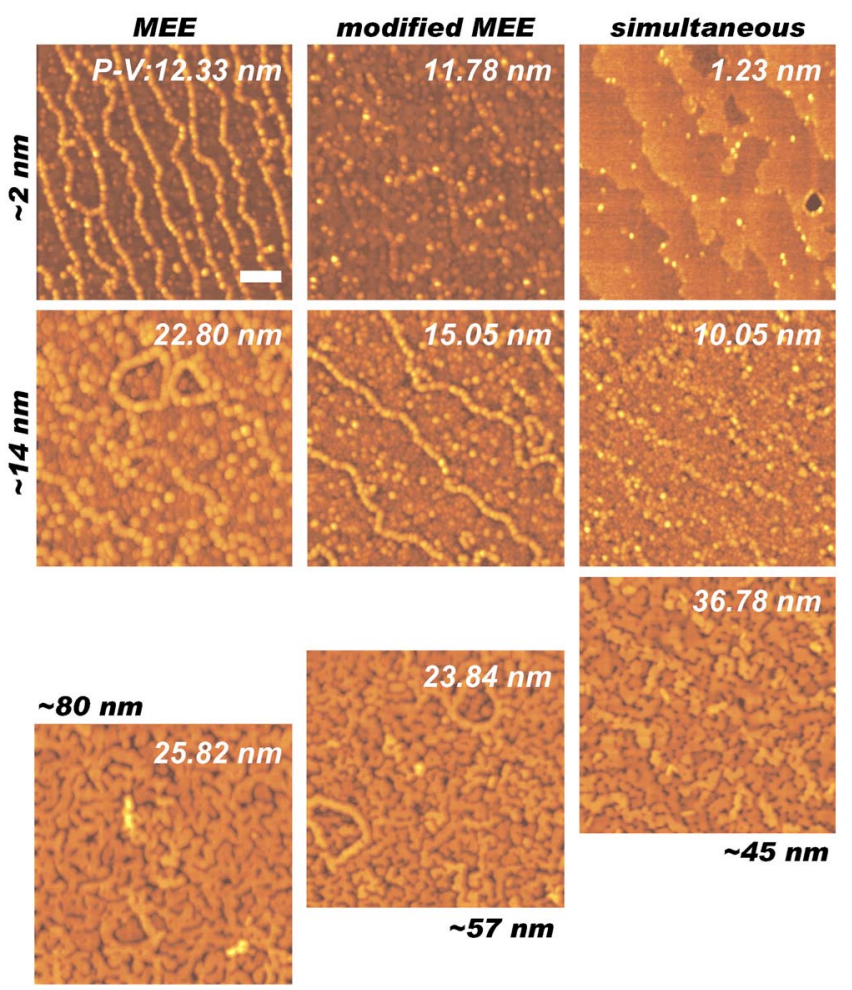

FIG. 2. (Color online) AFM images of AlN showing the evolution of initial nucleation. Thicknesses of $\sim 2$ and $\sim 14 \mathrm{~nm}$ are nominal. Left three, middle three, and right three images are for MEE, modified MEE, and simultaneous supply method, respectively. Scale bar represents $200 \mathrm{~nm}$.

dimensional growth due to the chemical and strain mismatch between sapphire and AlN.

However, differences due to the growth methods could be seen in their nucleation behavior. For example, comparing $\sim 14$-nm-thick AlN layers confirmed that the size of the 3D islands becomes larger in the order of simultaneous supply $(\phi: \sim 15 \mathrm{~nm})<$ modified $\operatorname{MEE}(\sim 25 \mathrm{~nm})<\operatorname{MEE}(\sim 50 \mathrm{~nm})$, suggesting that the degree of $\mathrm{Al}$ migration is in the same order. Considering this, let us compare two extreme cases, that is, MEE and the simultaneous supply method. For MEE, as clearly observed for the $\sim 2$-nm-thick AlN, initial nucleation occurs mostly along the step edges of the sapphire substrate, whereas for the simultaneous supply, random nucleation occurs both at the step edge and onto the terrace. These distinct initial nucleation behaviors are due to differences in their migration degrees, as schematically illustrated in Fig. 3. In MEE [Fig. 3(a)], migrating Al adatoms easily find sapphire step edges, which are the major nucleation sites, and are preferably adsorbed there. The sharp ridge structure indicates preferential adsorption of migrating $\mathrm{Al}$ adatoms onto already-existing AlN nuclei, probably due to the chemical matching between $\mathrm{Al}$ adatoms and $\mathrm{AlN}$. These ridges con- (a) MEE

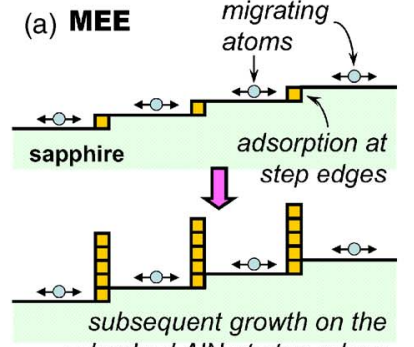

(b) modified MEE

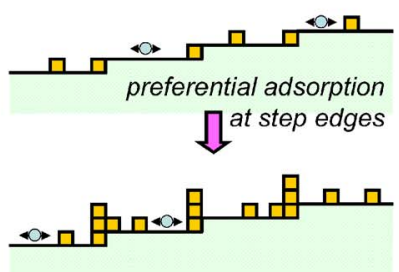

(c) simultaneous supply

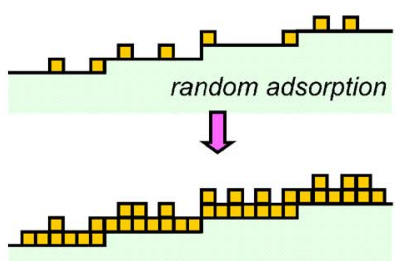

FIG. 3. (Color online) Schematic model of initial nucleation of AlN on sapphire. 


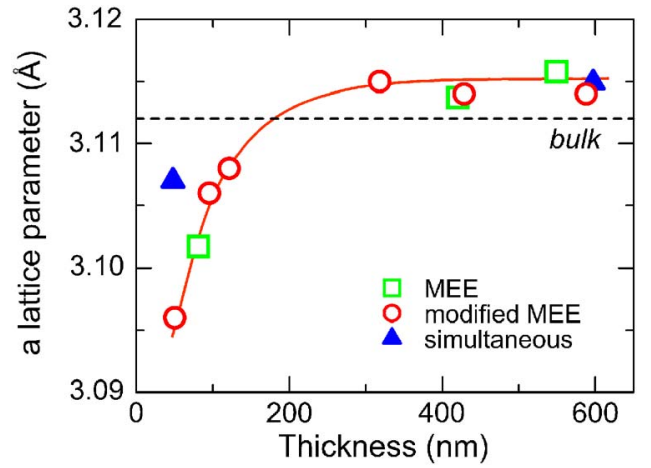

FIG. 4. (Color online) AlN a lattice parameter as a function of film thickness.

tinue to grow until nucleation, which also begins at the terrace, covers the sapphire surface. As shown in Fig. 2, the larger surface peak-to-valley values for thicker AlN are the consequence. On the contrary, random nucleation observed for the simultaneous supply method strongly suggests a negligible migration [Fig. 3(c)].

Based on the above discussion of the growth rates, the nucleation behavior during modified MEE should have intermediate characteristics of MEE and the simultaneous supply method. Al adatoms migrate well during the MEE mode in modified MEE in the presence of many nuclei formed during the simultaneous supply mode, which is macroscopically recognized as a smaller diffusivity of $\mathrm{Al}$ adatoms compared to MEE. In fact, similar to MEE, ridge structures are observed, but their height is lower than those formed by MEE as demonstrated in Fig. 2. Figure 3(b) schematically illustrates this nucleation model.

The ridge formation apparently roughened the surfaces, as evidenced by AFM presented in Fig. 2. Higher ridges should require thicker films in order to recover the surface flatness. As shown in Fig. 2, equivalent surface morphologies that show the coalescence of the initial nuclei are confirmed at $80 \mathrm{~nm}$ for MEE, $57 \mathrm{~nm}$ for modified MEE, and $45 \mathrm{~nm}$ for the simultaneous supply method. Further increasing the AlN thickness promoted coalescence, and eventually, as demonstrated in Fig. 1, generated a nearly perfectly flat surface at a thickness of $600 \mathrm{~nm}$ using modified MEE. The presence of pits in the MEE-grown AlN suggests that it is difficult to recover the surface flatness with this thickness.

To gain some insight into the influence of initial nucleation on the film quality, the lattice relaxation was assessed by XRD as a variation of the in-plane lattice parameter. Figure 4 shows the results. MEE and modified MEE traced the same tendency, and 50-nm-thick AlN involved $-0.52 \%$ compressive strain. On the other hand, $-0.18 \%$ compressive strain was measured in 48-nm-thick AlN grown by the simultaneous supply method. These findings indicate a slower strain relaxation in both modified MEE and MEE. Thus, the initial nucleation (Fig. 2) implies that the relatively uniform distribution of 3D nuclei by the simultaneous supply causes homogeneous lattice relaxation, while the ridge structures formed by MEE and modified MEE cause partial relaxation, possibly at the ridges. Hence, the difference in the lattice relaxation due to the growth methods may greatly affect the propagation of dislocations, and is currently under investigation.
For GaN MOVPE, LT-GaN has often been used as a nucleation layer because tiny GaN islands, which work as seeds for subsequent high-temperature growth, are formed only at low temperatures. On the contrary, for AlN, the tiny, densely packed nuclei were observed during the initial growth stage even for high-temperature growth, which is why nucleation layers are unnecessary for AlN. Furthermore, it is noteworthy that because modified MEE consists of MEE and the simultaneous supply method, the appropriate flow sequence may simultaneously control initial nucleation and the lattice relaxation processes. For example, increasing the occupation of the MEE mode within one cycle of modified MEE promotes the migration of $\mathrm{Al}$ adatoms to form higher ridge. Hence, this ability to control the initial growth may be advantageous over other methods.

Finally, we briefly refer to the optical property of the best-quality, modified-MEE-grown AIN. Photoluminescence (PL) measurements were performed at $10 \mathrm{~K}$ using an $\mathrm{ArF}$ laser (4 ns pulse with $50 \mathrm{~mJ} / \mathrm{cm}^{2}$ ). A peak was detected at $\sim 207 \mathrm{~nm}$, which is attributed to the overlap of free- and bound-excitonic emissions. The PL intensity increased in the off $c$-axis directions, as expected from the optical selection rule. Using the procedure proposed in Ref. 17, the out-ofplane polarization degree was evaluated to be $76 \%$, which may be an underestimation because of the total reflection within AlN and the solid angle of the detecting lens with a numerical aperture of 0.24 .

In summary, the initial nucleation and lattice relaxation in AlN grown by different growth methods were investigated. The Al-adatoms migration degree and the lattice relaxation mechanism led to the different surface morphologies and crystal qualities. The superior quality of AlN grown by modified MEE indicates the importance of controlling the initial nucleation to obtain high-quality AlN.

${ }^{1}$ T. Shibata, K. Asai, S. Sumiya, M. Mouri, M. Tanaka, O. Oda, H. Katsukawa, H. Miyake, and K. Hiramatsu, Phys. Status Solidi C 0, 2023 (2003).

${ }^{2}$ Q. Paduano and D. Weyburne, Jpn. J. Appl. Phys., Part 1 42, 1590 (2003). ${ }^{3}$ N. Onojima, J. Suda, and H. Matsunami, Jpn. J. Appl. Phys., Part 2 42, L445 (2003)

${ }^{4}$ J. F. Kaeding, Y. Wu, T. Fujii, R. Sharma, P. T. Fini, J. S. Speck, and S. Nakamura, J. Cryst. Growth 272, 257 (2004).

${ }^{5}$ Y. Taniyasu, M. Kasu, and T. Makimoto, Nature (London) 441, 325 (2006).

${ }^{6}$ K. Balakrishnan, A. Bandoh, M. Iwaya, S. Kamiyama, H. Amano, and I. Akasaki, Jpn. J. Appl. Phys., Part 2 46, L307 (2007).

${ }^{7}$ M. Kurimoto, T. Nakada, Y. Ishihara, M. Shibata, T. Honda, and H. Kawanishi, Jpn. J. Appl. Phys., Part 2 38, L551 (1999).

${ }^{8}$ M. Hiroki and N. Kobayashi, Jpn. J. Appl. Phys., Part 1 42, 2305 (2003).

${ }^{9}$ V. Adivarahan, W. H. Sun, A. Chitnis, M. Shatalov, S. Wu, H. P. Maruska, and M. A. Khan, Appl. Phys. Lett. 85, 2175 (2004).

${ }^{10}$ M. Takeuchi, H. Shimizu, R. Kajitani, K. Kawasaki, Y. Kumagai, A. Koukitu, and Y. Aoyagi, J. Cryst. Growth 298, 336 (2007).

${ }^{11}$ H. Hirayama, T. Yatabe, N. Noguchi, T. Ohashi, and N. Kamata, Appl. Phys. Lett. 91, 071901 (2007).

${ }^{12}$ J. P. Zhang, M. A. Khan, W. H. Sun, H. M. Wang, C. Q. Chen, Q. Fareed, E. Kuokstis, and J. W. Yang, Appl. Phys. Lett. 81, 4392 (2002).

${ }^{13}$ M. Takeuchi, S. Ooishi, T. Ohtsuka, T. Maegawa, T. Koyama, S. F. Chichibu, and Y. Aogi, Appl. Phys. Express 1, 021102 (2008).

${ }^{14}$ J. P. Zhang, M. A. Khan, H. M. Wang, W. H. Sun, V. Adivarahan, S. Wu, A. Chitnis, C. Q. Chen, M. Shatalov, E. Koukstis, J. W. Yang, and M. A. Khan, J. Electron. Mater. 32, 364 (2003).

${ }^{15}$ C. G. Dunn and E. F. Koch, Acta Metall. 5, 548 (1957).

${ }^{16}$ R. Di Felice and J. E. Northrup, Appl. Phys. Lett. 73, 936 (1998).

${ }^{17}$ Y. Taniyasu, M. Kasu, and T. Makimoto, Appl. Phys. Lett. 90, 261911 (2007). 